\title{
DOSIMETRIC COMPARISON OF HEART DOSE WITH 3DCRT VS IMRT IN POST-MASTECTOIMY LEFT SIDED CHEST WALL IRRADIATION
}

KEY WORDS: cardiac sparing, breast cancer, Intensity modulated radiotherapy.
Anushree Loyal*

Tapan Kapoor

\section{Jyoti Sharma}

Assistant Professor, Department of Radiation Oncology, Mahatma Gandhi Medical College,Jaipur. *Corresponding Author

Senior Resident, Department of Radiation Oncology, Mahatma Gandhi Medical College, Jaipur

Junior Resident, Department of Radiation Oncology, Mahatma Gandhi Medical College, Jaipur.

Purpose: Data regarding the efficacy of various radiotherapy techniques for post mastectomy chest-wall radiotherapy (PMRT) using hypofractionation is scarce and cardiac toxicity remains a concern. This study aims to compare effect of IMRT (Intensity Modulated Radiation Therapy) and 3D-CRT (3-Dimensional Conformal Radiation Therapy) techniques E. on cardiac dose.

Materials and Methods: In this retrospective study, we compared IMRT and 3DCRT plans of 20 patients who received PMRT to a dose of 42.56Gy/16\# and the dosimetric parameters in terms of planning target volume (PTV) coverage and dose to organ at risk (OARs) including heart and ipsilateral lung were recorded and analyzed.

Result: PTV coverage were comparable with both techniques. IMRT planning provided a better conformity index as

compared with 3DCRT ( 0.95 vs $0.91, \mathrm{p}<0.001)$. The mean dose to the heart significantly reduced with IMRT (4.36Gy vs 8.2Gy, p<0.00001).

Conclusion: IMRT offers a significant reduction in mean heart dose than 3DCRT in patients treated with hypofractionated post-mastectomy irradiation.

\section{INTRODUCTION:}

Female breast cancer has now surpassed lung cancer as the leading cause of global cancer incidence (11.7\%) in 2020 and is the fifth leading cause of cancer mortality worldwide ${ }^{1}$.

In India, breast cancer ranks as the leading cause of cancer in terms of incidence (13.5\%) and cancer death (10.6\%).

Radiotherapy is an integral part of multidisciplinary management of breast cancer.

Hypofractionated radiotherapy is the current standard for adjuvant whole breast radiotherapy ${ }^{2}$. For PMRT with hypofractionated radiotherapy, recently a phase III trial showed no differences in overall survival or late toxicities but higher grade 3 acute skin toxicity ( $8 \%$ vs. $3 \%)$. $^{3}$

One concern with PMRT is the potential risk of increasing mortality due to radiation-induced damage to myocardium. Over the last decade, awareness of cardiac morbidity during breast cancer radiotherapy has increased significantly. Two studies by Darby et al. ${ }^{4}$ and Sardaro et al. ${ }^{5}$ reported an increased risk of cardiac diseases by $7.4 \%$ and $4 \%$ for each gray (Gy) mean heart dose, respectively.

Various cardiac sparing techniques have been recommended like prone position or specific breathing techniques like deep-inspiration breath-hold (DIBH)/gating or by radiation techniques like IMRT or protons ${ }^{6}$, however, the recommendations are not yet clear ${ }^{7}$.

In light of the above evidence, this study was designed to compare the dosimetric parameters in terms of heart dose with two different radiotherapy techniques in postmastectomy patients treated with hypofractionated radiotherapy.

\section{MATERIAL AND METHODS:}

The present study is a retrospective dosimetric study, which was done in the department of radiation oncology, Mahatma Gandhi Medical College and Hospital, Jaipur, to investigate the impact on heart dose with IMRT versus 3DCRT.

20 patients with stage II-III, treated from July 2018-Dec 2021 , with PMRT were included in the study. Patients receiving RT to internal mammary node or with other dose fractionation were excluded. All the patients were treated with IMRT to left chest wall (CW) alone or left $\mathrm{CW}$ and left supraclavicular fossa(SCF). The treatment records and radiation planning details of patients treated with IMRT were retrieved. Since the anatomy of the chest wall is different for different patients and may impact heart dose, the same set of patients were planned with both the techniques (3DCRT and IMRT) and the doses were then compared.

All the patients had their simulation done as per the departmental protocol. The clinical target volume (CTV) and Planning Target Volume (PTV) was contoured according to the Radiation Therapy Oncology Group (RTOG) breast cancer atlas guidelines ${ }^{8}$. Organs at risk (OAR) contoured included heart, lung, spinal cord, oesophagus and contralateral breast. The heart was contoured as per the RTOG and cardiac contouring atlas ${ }^{9}$. All patients were planned on Eclipse TPS with a 6-MV beam.

IMRT plans for all the 20 patients were retrieved. IMRT plans were made using inverse planning with 5-7 beams. 3D-CRT plans for same 20 patients were generated using bitangential beams for chest wall and a direct field for SCF. Enhanced dynamic wedges and field in field techniques were used when required. A hypo-fractionated dose of $42.56 \mathrm{~Gy} / 16 \#$, @2.66Gy/\# was prescribed.

For dosimetric analysis, following parameters/indices were evaluated:

PTV: $D_{\text {near-max }}\left(D_{2 \%}\right), D_{\text {near-min }}\left(D_{98 \%}\right)$, Conformity index $\left(\mathrm{CI}_{\text {RTOG }}\right)$ (Volume of reference isodose/target volume), $\mathrm{V}_{107 \%}$

OARs:Lung: $\mathrm{V}_{5}, \mathrm{~V}_{20}$ and $\mathrm{D}_{\text {mean }}$

Heart: $\mathrm{V}_{5}, \mathrm{~V}_{25}$ and $\mathrm{D}_{\text {mean }}$

Opposite breast/lung: $\mathrm{D}_{\text {mean }}$

\section{STATISTICAL ANALYSIS:}

Data was analyzed using Statistical Package for Social Sciences (SPSS) version 27.0.1 (IBM Corp., Armonk, New York, USA). The t-test for two independent means was used for quantitative data. The $\mathrm{p}$-value reports were two-tailed and an alpha level of 0.05 was used to assess statistical significance. 


\section{RESULTS:}

Baseline patient and tumor characteristics are depicted in table 1. The mean age of the patient in our study was 50 years. $60 \%$ patients had stage III tumors.

Table 1: Baseline patient and tumor characteristics

\begin{tabular}{|l|l|}
\hline CHARACTERISTICS & NUMBER OF PATIENTS \\
\hline$\cdot \quad$ Age (yrs) & \\
\hline$<50$ & 09 \\
\hline$>50$ & 11 \\
\hline$\cdot \quad$ Menopausal Status & \\
\hline Pre-menopausal & 08 \\
\hline Postmenopausal & 12 \\
\hline$\cdot$ Tumor Stage & \\
\hline \multicolumn{1}{|c|}{ II } & 04 \\
\hline \multicolumn{1}{|c|}{ III } & 16 \\
\hline$\cdot \quad$ Chemotherapy & \\
\hline Neo-adjuvant & 05 \\
\hline Adjuvant & 15 \\
\hline
\end{tabular}

Dosimetric parameters for PTV and OARs are shown in table 2. The indices for PTV coverage were similar in both the arms. The mean dose to the heart was 4.36 Gy with IMRT while 8.2Gy with 3DCRT $(p<0.00001) . V_{25}$ heart was found to be significantly better with IMRT, however $\mathrm{V}_{5}$ did not show significant difference. For lung, $D_{\text {mean }}$ and $V_{20}$ exhibited significant dose reduction with IMRT. $\mathrm{V}_{5}$ of lung showed significant reduction with 3DCRT $(p<0.001)$. A wide dose range for lung dose were observed due to inclusion of two patients with CW irradiation only, in which the lung dose decreases as compared to CW plus SCF irradiation. $\mathrm{D}_{\text {mean }}$ to the contralateral lung was 0.86 Gy (IMRT) vs $0.92 \mathrm{~Gy}$ (3DCRT) and mean dose to the opposite breast were 1.13 Gy (IMRT) and $1.8 \mathrm{~Gy}(3 \mathrm{DCRT})$.

Table 2: Dosimetric parameters of PTV and OARs

\begin{tabular}{|l|l|l|l|}
\hline PTV & 3DCRT Group & IMRT Group & p-value \\
\hline $\mathrm{D}_{2}(\mathrm{~Gy})$ & $45.75(44.68-46.39)$ & $44.5(43.63-45.43)$ & 0.83 \\
\hline $\mathrm{D}_{98}(\mathrm{~Gy})$ & $37.87(34.44-40.6)$ & $\begin{array}{l}39.02(35.55- \\
41.09)\end{array}$ & 0.52 \\
\hline $\mathrm{D}_{\text {mean }}(\mathrm{Gy})$ & $42.2(41.82-44.56)$ & $\begin{array}{l}42.92(42.28- \\
43.87)\end{array}$ & 0.95 \\
\hline $\mathrm{V}_{107 \%}$ & $1.2(0.06-2.4)$ & $0.11(0.001-1.2)$ & 0.02 \\
\hline CI $_{\text {RTOG }}$ & $0.91(0.82-0.97)$ & $0.95(0.85-0.98)$ & $<0.001$ \\
\hline LUNG & & & \\
\hline $\mathrm{V}_{5 \%}$ & $45.68(25.2-60)$ & $55.35(33.9-80.0)$ & $<0.001$ \\
\hline $\mathrm{V}_{20 \%}$ & $33.2(18-38.2)$ & $26.74(16.2-37.1)$ & $<0.001$ \\
\hline $\mathrm{D}_{\text {mean }}(\mathrm{Gy})$ & $17.08(12.68-$ & $13.05(10.35-$ & $<0.001$ \\
\hline HEART & $20.26)$ & $16.62)$ & \\
\hline $\mathrm{V}_{5 \%}$ & $21.6(8.78-32.6)$ & $23.32(12.7-40.0)$ & 0.2 \\
\hline $\mathrm{V}_{25 \%}$ & $9.6(5.1-13.8)$ & $5.70(1.8-11.3)$ & $<0.001$ \\
\hline$D_{\text {mean }}(\mathrm{Gy})$ & $8.2(6.6-9.1)$ & $4.36(3.9-6.73)$ & $<0.00001$ \\
\hline
\end{tabular}

\section{DISCUSSION:}

There is no robust evidence on the optimal technique for cardiac sparing in left-sided breast carcinoma patients treated with hypofractionated radiotherapy. Newer techniques have shown some promise in reducing the heart dose. However, the cost of such treatment is high, about 2.5 times with IMRT and even higher with protons, which poses a significant economic burden on the patients, especially in the Indian setting. Also, these new techniques with gating are not readily available. Conventional methods to reduce heart dose like prone position is also recommended, however, literature data are not univocal ${ }^{10}$. Also, the prone position is associated with a worse setup accuracy.

In this study, when the targeted volumes receiving $D_{2 \%}$ and $\mathrm{D}_{98 \%}$ of prescribed PTV dose were compared, there was no significant difference between IMRT and 3DCRT which suggest that both are comparable in terms of coverage. In a similar study ${ }^{11}$, of conventionally fractionated RT, the coverage was similar. On comparing the $\mathrm{V}_{107 \%}$ of PTV, IMRT plans were significantly better $(\mathrm{p}<0.02)$. High dose volumes with 3DCRT plans were more (1.2\%), however within acceptable limits ${ }^{12}$.

On comparing the conformity Index $\left(\mathrm{CI}_{\text {RTOG }}\right)$, the IMRT plans were significantly superior. Certain studies have showed significant improvement in CI while using IMRT with PTV coverage ranging from $90-97 \%$ of the prescribed dose which is consistent with the results of our present study (IMRT $0.95 \mathrm{v}$ 3DCRT $0.91 \mathrm{~s}, \mathrm{p}<0.001)^{13}$. Whereas Li et al. deduced that use of IMRT technique was not associated with significant improvement in CI. ${ }^{14}$

In the present study, the mean to heart with IMRT and 3DCRT were 4.36 and 8.2 Gy respectively $(p<0.00001)$. The $V_{25}$ for IMRT and 3DCRT were 5.7 and 9.6Gy $(p<0.001)$. The low dose volume $\mathrm{V}_{5}$ however were similar with both the techniques. The literature on same is controversial ${ }^{6,11}$. This suggest that the IMRT plans were superior to 3DCRT in reducing the dose to heart.This data is in accordance with other studies from past ${ }^{15}$, while a few others and our study have shown either equivalent or better results with the 3D-CRT technique ${ }^{11,16}$

Advances in RT techniques have been shown to reduce the incidence of radiation pneumonitis. Techniques designed to reduce the heart dose have also significantly decreased the dose to the lung by more than $50 \% .^{17}$

For lung, $\mathrm{V}_{20}$ (i.e. the volume of the lung receiving 20Gy) and mean dose denotes the risk of radiation pneumonitis. In the present study, the mean lung dose was significantly lower with IMRT (13.05 vs 17.08, $\mathrm{p}<0.001$ ) and so was $\mathrm{V}_{20}$. Kahan and Li et al. concluded that PMRT using IMRT significantly decreased $\mathrm{V}_{20}$ and $\mathrm{V}_{30}$ of the ipsilateral lung as compared to 3DCRT. (29\% $+/-2 \%$ and $21 \%+/-2 \%$ in IMRT arm vs $32 \%+/-6 \%$ and $22+/-$ $5 \%$ in 3DCRT arm $)^{14}$. The low dose volume $\left(\mathrm{V}_{5}\right)$ was however more with IMRT. 3DCRT plans were able to control low dose volumes better.

To summarize, a marked improvement in the reduction of mean heart dose and mean lung dose was seen with IMRT plans as compared with 3DCRT plans with similar coverage of the target volumes. IMRT techniques delivered plans with more conformity and its use to reduce the heart dose, also decreased the lung dose.

\section{REFERENCES}

1. Hyuna Sung, Jacques Ferlay, Rebecca L. Siegel et al. Global Cancer Statistics 2020: GLOBOCAN Estimates of Incidence and Mortality Worldwide for 36 Cancers in 185 Countries.CA CANCER J CLIN 2021;71:209-249

2. Luca F Valle, Surbhi Agarwal et al. Hypofractionated whole breast radiotherapy in breast conservation for early-stage breast cancer: a systematic review and meta-analysis of randomized trials. Breast Cancer Res Treat.2017;162(3):409-417.

3. Shu-Lian Wang, Hui Fang et al. Hypofractionated versus conventional fractionated postmastectomy radiotherapy for patients with high-risk breast cancer: a randomised, non-inferiority, open-label, phase 3 trial. Lancet Oncology 2019 Mar;20(3):352-360.

4. Sarah C Darby, Marianne Ewertz et al. Risk of ischemic heart disease in women after radiotherapy for breast cancer. N Engl J Med 2013 Mar 14;368(11):987-98.

5. Sardaro A, Petruzzelli MF et al, Radiation-induced cardiac damage in early left breast cancer patients: Risk factors, biological mechanisms, radiobiology, and dosimetric constraints. Radiother Oncol 103(2): 133-142.

6. Marciana-Nona Duma, René Baumann et.al. Heart-sparing radiotherapy techniques in breast cancer patients: a recommendation of the breast cancer expert panel of the German society of radiation oncology (DEGRO). Strahlenther Onkol.2019 Oct;195(10):861-871.

7. Munshi Anusheel, Khataniar et al. (2018). Spatial orientation of coronary arteries and its implication for breast and thoracic radiotherapy-proposing "coronary strip" as a new organ at risk. Strahlentherapie und Onkologie. 194. 10.1007/s00066-018-1299-x.

8. Tony Mathew, Michael Chao, Carminia Lapuz et al. Consistency of ESTRO and RTOG contouring guidelines for target volume delineation in early-stage breast cancer. Int J Radiol Radiat Ther. 2020;7(5):133 140.

9. Frances Duane, Marianne C. Aznar et al. A cardiac contouring atlas for radiotherapy. Radiotherapy and Oncology 122 (2017) 416-422.

10. Varga $\mathrm{Z}$, Hideghéty $\mathrm{K}$ et al. Individual positioning: a comparative study of adjuvant breast radiotherapy in the prone versus supine position. Int J Radiat Oncol Biol Phys. 2009 Sep 1;75(1):94-100.

11. Rastogi K, Sharma S et al. Dosimetric comparison of IMRT versus 3DCRT for 
post-mastectomy chest wall irradiation. Radiat Oncol J.2018 Mar;36(1):71-78.

12. M.S.Thomsen, M. Berg et al.Dose constraints for whole breast radiation therapy based on the quality assessment of treatment plans in the randomised Danish breast cancer group (DBCG) HYPO trial, Clinical and Translational Radiation breast cancer group (DBCG) HYPO trial, Clinical and Tran
Oncology,Volume 28,2021,Pages 118-123,ISSN 2405-6308,

13. Zhou GX, Xu SP, Dai XK, et al. Clinical dosimetric study of three radiotherapy techniques for postoperative breast cancer: Helical Tomotherapy, IMRT, and 3D-CRT.Technol Cancer Res Treat 201 1;10:15-23.

14. Li W, Wang J, Cheng H, Yu H, Ma J. IMRT versus 3D-CRT for post-mastectomy irradiation of chest wall and regional nodes: a population-based comparison of normallung dose and radiation pneumonitis.IntJ ClinExpMed 2016;9:22331-7 .

15. Menzel H-G. International Commission on Radiation Units and Measurements. Journal of the ICRU.2015;15(1-2):1-2.doi:10.1093/jicru ndy 011

16. Mehta A, Kumar P et al. Dosimetric parameters of heart and left ventricle comparison of 3D CRT and IMRT in left cancer breast. Int J Med Res Rev. 2019;7(6):468-474

17. Lee BM, Chang JS et al. Hypofractionated Radiotherapy Dose Scheme and Application of New Techniques Are Associated to a Lower Incidence of Radiation Pneumonitis in Breast Cancer Patients.Front Oncol.2020 Feb 1 1;10:124. 